\title{
Microscopic construction of the two-fluid model for superfluid helium-4
}

\author{
P. Shygorin, A. Svidzynskyj \\ Volyn National University of Lesia Ukrainka, Lutsk 43025, Avenue Voli, 13, Ukraine
}

Received June 24, 2009, in final form September 30, 2009

\begin{abstract}
Using a system of Heisenberg's equation of motion for both the normal and the anomalous correlation functions a two-fluid hydrodynamics for superfluid helium- 4 was constructed. The method is based on a gradient expansion of the exact equations of motion for correlation functions about a local equilibrium together with explicit use of the local equilibrium statistical operator for superfluid helium in the frame of reference, where condensate is in the state of rest.
\end{abstract}

Key words: two-fluid hydrodynamics, correlation function, superfluid helium, statistical operator

PACS: $67.25 . d g$

\section{Introduction}

Superfluid behavior is the most striking property of liquid helium-4. The superfluid ${ }^{4} \mathrm{He}$ is a quantum degenerate system with spontaneously broken symmetry. Its feature is the macroscopic occupation of the lowest-energy single-particle quantum state or, in other words, it is the presence of condensate. As a result, the state of statistical equilibrium of the system with spontaneously broken symmetry depends on eight quantities: particle density $\rho$, energy density $\varepsilon$, momentum density $\vec{j}$ and superfluid velocity $\vec{v}_{\mathrm{s}}$. Presence of additional velocity field leads to the two-fluid hydrodynamics of such a system.

The two-fluid hydrodynamic equations for the superfluid ${ }^{4} \mathrm{He}$ in the phenomenological consideration were constructed by Landau in 1941 [1]. These equations were derived at the microscopic level by Bogolyubov in 1963 [2].

As a starting point in Bogolyubov's paper, there is a set of equations of motion for local quantities (particle density, momentum density and energy density), which easily follows from Heisenberg equations for both creation and annihilation operators; as well as the equation for anomalous average $\langle\psi\rangle$. Thus, from the latter there follows a hydrodynamic equation for superfluid velocity.

To perform a transition from formal equations of motion to hydrodynamic equations Bogolyubov considered a stage of evolution when it approaches the equilibrium. Then it is possible to assume that a local equilibrium is established in the system. It is described by the statistical operator with parameters that depend on space coordinates. At the approach to the thermodynamic equilibrium these parameters are slowly changed in space and time and therefore their gradients are small. The procedure of expansion by the gradients is formulated by the introduction of the so-called "parameter of homogeneity" into the equations of motion. Then, expansion in terms of gradients coincides with expansion by this parameter. Introduction of the parameter of homogeneity in Bogolyubov's paper was carried out in a formal way.

When the conservation relations for the local hydrodynamical quantities are constructed, the next step is to calculate the hydrodynamical flows in these equations. Bogolyubov calculated the momentum flux by using a very elegant "scale transformation" method. But the flux of energy is obtained inconsistently. A more acceptable method of calculation of the energy flux using an explicit local equilibrium statistical operator was proposed by Morozov [3]. 
Our paper is similar to the Bogolyubov's article [2], but we work with equations of motion for the correlation functions which are written in the mixed Wigner representation. It permits an expansion by the gradients to be directly realized with great ease and with rigorous mathematics.

To calculate hydrodynamic flows we use an explicit form for the local equilibrium statistical operator. But in contrast to Morozov's work, which operates with statistical operator of the superfluid helium at the laboratory reference system, we construct one at the reference system in which the condensate is motionless. This provides an essential simplification, because in the local frame of reference moving with $\vec{v}_{\mathrm{s}}$ the superfluid component is stopped, then the total current is carried by the normal component.

We conditionally separate the construction of the two-fluid model into two stages. At the first stage, using Heisenberg equation of motion for both normal and anomalous correlation functions, we derive conservation relations for densities of particle $\rho$, momentum $\vec{j}$ and energy $\varepsilon$, as well as equation of motion for superfluid velocity $\vec{v}_{\mathrm{s}}$. At the second stage, we express hydrodynamic flows in conservation relations in terms of the already introduced variables $(\rho, \vec{j}$ and $\varepsilon)$.

\section{Construction of the two-fluid hydrodynamic equations}

\subsection{Equation of motion for correlation functions in the mixed Wigner representation}

The helium-4 is a typical Bose system with pair interaction. Its Hamiltonian in the second quantization representation has the following form (we set $\hbar=1$ throughout this paper)

$$
H=\int \mathrm{d} \vec{r} \psi^{+}(\vec{r})\left(-\frac{1}{2 m} \Delta\right) \psi(\vec{r})+\frac{1}{2} \int \mathrm{d} \vec{r} \mathrm{~d} \vec{r}^{\prime} \Phi\left(\vec{r}-\vec{r}^{\prime}\right) \psi^{+}(\vec{r}) \psi^{+}\left(\vec{r}^{\prime}\right) \psi\left(\vec{r}^{\prime}\right) \psi(\vec{r})
$$

here $\psi^{+}(\vec{r})$ and $\psi(\vec{r})$ - are the creation and annihilation operators, respectively, $\Phi\left(\vec{r}-\vec{r}^{\prime}\right)=$ $\Phi\left(\left|\vec{r}-\vec{r}^{\prime}\right|\right)$ - is interaction potential.

To construct the hydrodynamics of a system with spontaneously broken symmetry we should proceed from the extended system of correlation functions [5], which is formed both by a normal and by an anomalous correlation function. Therefore, we will start with the system correlation functions in the next form

$$
\left\langle\psi^{+}\left(\vec{r}_{1}, t\right) \psi\left(\vec{r}_{2}, t\right)\right\rangle, \quad\langle\psi(\vec{r}, t)\rangle .
$$

Here, the angular brackets indicate an average at the local-equilibrium ensemble, and the dependence of the creation and annihilation operators on the time is given through a Heisenberg representation, for instance

$$
\psi(\vec{r}, t)=\mathrm{e}^{\mathrm{i} H t} \psi(\vec{r}) \mathrm{e}^{-\mathrm{i} H t}
$$

Notice, that an average in (2) is treated as quasi-average [4]. For the sake of simplicity, we will not take " $\nu$ "-term into account which breaks the symmetry of the Hamiltonian (1).

Using the Heisenberg's equation of motion

$$
\mathrm{i} \frac{\partial \psi(\vec{r}, t)}{\partial t}=[\psi(\vec{r}, t), H]_{-}=-\frac{1}{2 m} \Delta \psi(\vec{r}, t)+\int \mathrm{d} \vec{r}^{\prime} \Phi\left(\vec{r}-\vec{r}^{\prime}\right) \psi^{+}\left(\vec{r}^{\prime}, t\right) \psi\left(\vec{r}^{\prime}, t\right) \psi(\vec{r}, t)
$$

we obtain the equations of motion for correlation functions (2).

These equations are as follows

$$
\begin{aligned}
& \mathrm{i} \frac{\partial}{\partial t}\left\langle\psi^{+}\left(\vec{r}_{1}, t\right) \psi\left(\vec{r}_{2}, t\right)\right\rangle=\frac{1}{2 m}\left(\Delta_{1}-\Delta_{2}\right)\left\langle\psi^{+}\left(\vec{r}_{1}, t\right) \psi\left(\vec{r}_{2}, t\right)\right\rangle \\
& -\int \mathrm{d} \vec{r}^{\prime}\left\{\Phi\left(\vec{r}_{1}-\vec{r}^{\prime}\right)-\Phi\left(\vec{r}_{2}-\vec{r}^{\prime}\right)\right\}\left\langle\psi^{+}\left(\vec{r}_{1}, t\right) \psi^{+}\left(\vec{r}^{\prime}, t\right) \psi\left(\vec{r}^{\prime}, t\right) \psi\left(\vec{r}_{2}, t\right)\right\rangle, \\
& \mathrm{i} \frac{\partial}{\partial t}\langle\psi(\vec{r}, t)\rangle=-\frac{1}{2 m} \Delta\langle\psi(\vec{r}, t)\rangle+\int \mathrm{d} \vec{r}^{\prime} \Phi\left(\vec{r}-\vec{r}^{\prime}\right)\left\langle\psi^{+}\left(\vec{r}^{\prime}, t\right) \psi\left(\vec{r}^{\prime}, t\right) \psi(\vec{r}, t)\right\rangle .
\end{aligned}
$$


The next step will be the separation of gauge-noninvariant multipliers (in fact we will use a reference system in which the condensate is motionless). Such a separation of the phase has the form

$$
\psi(\vec{r}, t) \rightarrow \tilde{\psi}(\vec{r}, t)=\psi(\vec{r}, t) \mathrm{e}^{\mathrm{i} m \chi(\vec{r}, t)}
$$

The separation of the phase transforms correlation functions following the rules

$$
\begin{aligned}
\left\langle\psi^{+}\left(\vec{r}_{1}, t\right) \psi\left(\vec{r}_{2}, t\right)\right\rangle & =\mathrm{e}^{\mathrm{i} m\left(\chi\left(\vec{r}_{2}, t\right)-\chi\left(\vec{r}_{1}, t\right)\right)} G\left(\vec{r}_{1}, \vec{r}_{2} ; t\right), \\
\langle\psi(\vec{r}, t)\rangle & =\mathrm{e}^{\mathrm{i} m \chi(\vec{r}, t)} F(\vec{r} ; t) \\
\left\langle\psi^{+}\left(\vec{r}_{1}, t\right) \psi^{+}\left(\vec{r}^{\prime}, t\right) \psi\left(\vec{r}^{\prime}, t\right) \psi\left(\vec{r}_{2}, t\right)\right\rangle & =\mathrm{e}^{\mathrm{i} m\left(\chi\left(\vec{r}_{2}, t\right)-\chi\left(\vec{r}_{1}, t\right)\right)} \mathcal{D}^{(1)}\left(\vec{r}_{1}, \vec{r}_{2}, \vec{r}^{\prime} ; t\right), \\
\left\langle\psi^{+}\left(\vec{r}^{\prime}, t\right) \psi\left(\vec{r}^{\prime}, t\right) \psi(\vec{r}, t)\right\rangle & =\mathrm{e}^{\mathrm{i} m \chi(\vec{r}, t)} \mathcal{D}^{(2)}\left(\vec{r}, \vec{r}^{\prime} ; t\right) .
\end{aligned}
$$

The functions $G, F, \mathcal{D}^{(1)}$ and $\mathcal{D}^{(2)}$ at the statistical equilibrium state are spatially homogeneous. At the nonequilibrium states, the functions' changes will be spatially inhomogeneous.

Then, the equations of motion for $G$ and $F$ are as follows

$$
\begin{aligned}
& \left\{\mathrm{i} \frac{\partial}{\partial t}+m \dot{\chi}\left(\vec{r}_{1}, t\right)-m \dot{\chi}\left(\vec{r}_{2}, t\right)\right\} G\left(\vec{r}_{1}, \vec{r}_{2} ; t\right) \\
& =-\frac{1}{2 m}\left[\left(\hat{\vec{p}}_{1}-m \vec{v}_{\mathrm{s}}\left(\vec{r}_{1}, t\right)\right)^{2}-\left(\hat{\vec{p}}_{2}+m \vec{v}_{\mathrm{s}}\left(\vec{r}_{2}, t\right)\right)^{2}\right] G\left(\vec{r}_{1}, \vec{r}_{2} ; t\right) \\
& \quad-\int \mathrm{d} \vec{r}^{\prime}\left\{\Phi\left(\vec{r}_{1}-\vec{r}^{\prime}\right)-\Phi\left(\vec{r}_{2}-\vec{r}^{\prime}\right)\right\} \mathcal{D}^{(1)}\left(\vec{r}_{1}, \vec{r}_{2}, \vec{r}^{\prime} ; t\right) \\
& \left\{\mathrm{i} \frac{\partial}{\partial t}-m \dot{\chi}(\vec{r}, t)\right\} F(\vec{r} ; t)=\frac{1}{2 m}\left(\hat{\vec{p}}+m \vec{v}_{\mathrm{s}}(\vec{r}, t)\right)^{2} F(\vec{r} ; t) \\
& \quad+\int \mathrm{d} \vec{r}^{\prime} \Phi\left(\vec{r}-\vec{r}^{\prime}\right) \mathcal{D}^{(2)}\left(\vec{r}, \vec{r}^{\prime} ; t\right)
\end{aligned}
$$

where $\vec{v}_{\mathrm{s}}=\nabla \chi$ is superfluid velocity (velocity of the condensate).

The transition to equations of hydrodynamics is performed using an expansion of equation (5) in terms of space gradients. This expansion can be simply performed by using the so-called mixed Wigner representation [5]. For this purpose, we introduce new variables

$$
\vec{R}=\frac{1}{2}\left(\vec{r}_{1}+\vec{r}_{2}\right), \quad \vec{r}=\vec{r}_{2}-\vec{r}_{1}
$$

After the Fourier transformation with respect to relative coordinate $\vec{r}$ we obtain

$$
f\left(\vec{r}_{1}, \vec{r}_{2}, t\right) \rightarrow f(\vec{R}, \vec{r}, t)=\int \frac{\mathrm{d} \vec{p}}{(2 \pi)^{3}} f(\vec{R}, \vec{p}, t) \mathrm{e}^{\mathrm{i} \vec{p} \vec{r}}
$$

and

$$
\begin{array}{cc}
\vec{r}_{1} \rightarrow \vec{R}-\frac{\mathrm{i}}{2} \nabla_{\vec{p}}, & \vec{r}_{2} \rightarrow \vec{R}+\frac{\mathrm{i}}{2} \nabla_{\vec{p}}, \\
\hat{\vec{p}} \rightarrow \vec{p}-\frac{\mathrm{i}}{2} \nabla_{\vec{R}}, & \hat{\vec{p}} \rightarrow-\vec{p}-\frac{\mathrm{i}}{2} \nabla_{\vec{R}} .
\end{array}
$$

Any function of $\vec{R}+\mathrm{i} / 2 \cdot \nabla_{\vec{p}}$ can be understood in terms of its power-series expansion

$$
f\left(\vec{R}+\frac{\mathrm{i}}{2} \nabla_{\vec{p}}\right)=f(\vec{R})+\frac{\mathrm{i}}{2} \frac{\partial f(\vec{R})}{\partial \vec{R}} \frac{\partial}{\partial \vec{p}}-\cdots
$$


Using procedures (7) and (8) the equations for correlation functions can be written as follows

$$
\begin{aligned}
& \frac{\partial G_{\vec{p}}(\vec{R}, t)}{\partial t}-m \dot{v}_{s i}(\vec{R}, t) \frac{\partial G_{\vec{p}}(\vec{R}, t)}{\partial p_{i}}-\frac{\partial}{\partial R_{j}}\left(\frac{\left(p_{i}+m v_{s i}(\vec{R}, t)\right)^{2}}{2 m}\right) \frac{\partial G_{\vec{p}}(\vec{R}, t)}{\partial p_{j}} \\
& \quad+\left(\frac{p_{i}}{m}+v_{s i}(\vec{R}, t)\right) \frac{\partial G_{\vec{p}}(\vec{R}, t)}{\partial R_{i}}+\frac{\partial}{\partial R_{j}}\left(\frac{1}{2} \int \mathrm{d} \vec{r}^{\prime} \frac{\partial \Phi\left(r^{\prime}\right)}{\partial r_{i}^{\prime}} r_{j}^{\prime} \frac{\partial \mathcal{D}_{\vec{p}}^{(1)}\left(\vec{R}, \vec{r}^{\prime} ; t\right)}{\partial p_{i}}\right)=0 \\
& \left(\mathrm{i} \frac{\partial}{\partial t}-m \dot{\chi}(\vec{R}, t)\right) F(\vec{R}, t)=\frac{1}{2 m}\left(\hat{\vec{p}}+m \vec{v}_{s}^{2}(\vec{R}, t)\right) F(\vec{R}, t) \\
& -\mathrm{i}\left(\nabla_{\vec{R}} v_{s i}(\vec{R}, t)\right) F(\vec{R}, t)+\int \mathrm{d} \vec{r}^{\prime} \Phi\left(\left|\vec{R}-\vec{r}^{\prime}\right|\right) \mathcal{D}^{(2)}\left(\vec{R}, \vec{r}^{\prime} ; t\right)
\end{aligned}
$$

Here

$$
\begin{aligned}
G_{\vec{p}}(\vec{R}, t) & =\int \mathrm{d} \vec{r}\left\langle\psi^{+}\left(\vec{R}-\frac{\vec{r}}{2}, t\right) \psi\left(\vec{R}+\frac{\vec{r}}{2}, t\right)\right\rangle \mathrm{e}^{\mathrm{i} \vec{p} \vec{r}} \\
\mathcal{D}_{\vec{p}}^{(1)}\left(\vec{R}, \vec{r}^{\prime} ; t\right) & =\int \mathrm{d} \vec{r}\left\langle\psi^{+}\left(\vec{R}-\frac{\vec{r}}{2}, t\right) \psi^{+}\left(\vec{r}^{\prime}, t\right) \psi\left(\vec{r}^{\prime}, t\right) \psi\left(\vec{R}+\frac{\vec{r}}{2}, t\right)\right\rangle \mathrm{e}^{\mathrm{i} \vec{p} \vec{r}}
\end{aligned}
$$

In the obtained equation (9) the second order terms with respect to space gradient (the terms proportional to $\nabla_{\vec{R}}^{2}$ ) were neglected.

The equation (9) we denominate as a generating equation, because its application provides conservation laws for the hydrodynamic quantities. Equation of motion for superfluid velocity will be obtained in terms of (9).

Let us proceed to obtaining differential conservation laws (balance equations).

\subsection{Equation of motion for superfluid velocity}

Let us consider the equation for an anomalous correlation function (10). After separating real and imaginary parts in this equation we find

$$
\left(m \dot{\chi}(\vec{R}, t)+\frac{1}{2} m \vec{v}_{s}^{2}(\vec{R}, t)\right) F(\vec{R}, t)=\frac{1}{2 m} \nabla_{\vec{R}}^{2} F(\vec{R}, t)-\int \mathrm{d} \vec{r}^{\prime} \Phi\left(\left|\vec{R}-\vec{r}^{\prime}\right|\right) \mathcal{D}^{(2)}\left(\vec{R}, \vec{r}^{\prime} ; t\right) .
$$

Hence

or

$$
\dot{\chi}(\vec{R}, t)=-\frac{1}{2} \vec{v}_{\mathrm{s}}^{2}(\vec{R}, t)+\frac{\nabla_{\vec{R}}^{2} F(\vec{R}, t)}{2 m^{2} F(\vec{R}, t)}-\frac{\int \mathrm{d} \vec{r}^{\prime} \Phi\left(\left|\vec{R}-\vec{r}^{\prime}\right|\right) \mathcal{D}^{(2)}\left(\vec{R}, \vec{r}^{\prime} ; t\right)}{m F(\vec{R}, t)},
$$

Here

$$
\dot{\chi}(\vec{R}, t)=-\frac{1}{2} \vec{v}_{\mathrm{s}}^{2}(\vec{R}, t)-\frac{\mu(\vec{R}, t)}{m} .
$$

$$
\mu(\vec{R}, t)=-\frac{\nabla_{\vec{R}}^{2} F(\vec{R}, t)}{2 m^{2} F(\vec{R}, t)}+\frac{\int \mathrm{d} \vec{r}^{\prime} \Phi\left(\left|\vec{R}-\vec{r}^{\prime}\right|\right) \mathcal{D}^{(2)}\left(\vec{R}, \vec{r}^{\prime} ; t\right)}{m F(\vec{R}, t)}
$$

is chemical potential [2].

Applying the operation $\nabla_{\vec{R}}$ to equation (14) we obtain the equation of motion for superfluid velocity

$$
m \frac{\partial \vec{v}_{\mathrm{s}}}{\partial t}+\nabla_{\vec{R}}\left(\frac{m \vec{v}_{\mathrm{s}}^{2}}{2}+\mu\right)=0
$$

This is the first hydrodynamic equation which shows that the superfluid accelerates freely under the applied fields. The remaining hydrodynamic equations are provided by the conservation relations for the particle density $\rho(\vec{R}, t)$, momentum density $\vec{j}(\vec{R}, t)$ and energy density $\mathcal{E}(\vec{R}, t)$. These equations are simply obtained from calculation of moments of the generating equation (9). 


\subsection{Equation for particle density}

By definition

$$
\rho(\vec{R}, t)=m\left\langle\psi^{+}(\vec{R}, t) \psi(\vec{R}, t)\right\rangle=m \int \frac{\mathrm{d} \vec{p}}{(2 \pi)^{3}} G_{\vec{p}}(\vec{R}, t) .
$$

After integrating (9) over $\vec{p}$ we find

$$
\frac{\partial \rho}{\partial t}+\operatorname{div} \vec{j}=0
$$

where

$$
\vec{j}(\vec{R}, t)=\int \frac{\mathrm{d} \vec{p}}{(2 \pi)^{3}} \vec{p} G_{\vec{p}}(\vec{R}, t)+\rho \vec{v}_{\mathrm{s}} \equiv \vec{j}_{0}+\rho \vec{v}_{\mathrm{s}} .
$$

The (16) is an equation of continuity for particle density. The $\vec{j}(\vec{R}, t)$ is a momentum density, respectively, and $\vec{j}_{0}$ is a momentum density in the reference system where condensate is motionless. Calculation of the $\vec{j}_{0}$ in an explicit form will be performed in section 3 .

\subsection{Equation for momentum density}

Using definition (17), we find that

$$
\frac{\partial j_{k}}{\partial t}=\frac{\partial}{\partial t}\left(j_{0 k}+\rho v_{s k}\right)=\int \frac{\mathrm{d} \vec{p}}{(2 \pi)^{3}}\left(p_{k}+m v_{s k}\right) \frac{\partial G_{\vec{p}}(\vec{R}, t)}{\partial t}+\rho \frac{\partial v_{s k}}{\partial t}
$$

Taking the moment of the generating equation (9) with respect to $\vec{p}+m \vec{v}_{\mathrm{s}}$ and using the equation of motion for the superfluid velocity (15), we obtain

$$
\frac{\partial j_{k}}{\partial t}+\frac{\partial \Pi_{k j}}{\partial R_{j}}=0
$$

The flow of momentum density (stress tensor) is given by

$$
\begin{aligned}
\Pi_{k j} & =\frac{1}{m} \int \frac{\mathrm{d} \vec{p}}{(2 \pi)^{3}}\left(p_{k}+m v_{s k}\right)\left(p_{j}+m v_{s j}\right) G_{\vec{p}}(\vec{R}, t)-\frac{1}{2} \int \mathrm{d} \vec{r}^{\prime} \frac{\partial \Phi\left(r^{\prime}\right)}{\partial r_{k}^{\prime}} r_{j}^{\prime} \int \frac{\mathrm{d} \vec{p}}{(2 \pi)^{3}} \mathcal{D}_{\vec{p}}^{(1)}\left(\vec{R}, \vec{r}^{\prime} ; t\right) \\
& =v_{s k} j_{0 j}+v_{s j} j_{0 k}+\rho v_{s k} v_{s j}+\Pi_{0 k j}
\end{aligned}
$$

where

$$
\Pi_{0 k j}=\frac{1}{m} \int \frac{\mathrm{d} \vec{p}}{(2 \pi)^{3}} p_{k} p_{j} G_{\vec{p}}(\vec{R}, t)-\frac{1}{2} \int \mathrm{d} \vec{r}^{\prime} \frac{\partial \Phi\left(r^{\prime}\right)}{\partial r_{k}^{\prime}} r_{j}^{\prime} \int \frac{\mathrm{d} \vec{p}}{(2 \pi)^{3}} \mathcal{D}_{\vec{p}}^{(1)}\left(\vec{R}, \vec{r}^{\prime} ; t\right)
$$

\subsection{Equation for energy density}

By definition the energy density of particles in the laboratory system of reference is as follows

$$
\mathcal{E}(\vec{r}, t)=\frac{1}{2 m}\left\langle\nabla \psi^{+}(\vec{r}, t) \nabla \psi(\vec{r}, t)\right\rangle+\frac{1}{2} \int \mathrm{d} \vec{r}^{\prime} \Phi\left(\left|\vec{r}-\vec{r}^{\prime}\right|\right)\left\langle\psi^{+}(\vec{r}, t) \psi^{+}\left(\vec{r}^{\prime}, t\right) \psi\left(\vec{r}^{\prime}, t\right) \psi(\vec{r}, t)\right\rangle .
$$

In the system of reference where condensate is motionless the energy density is

$$
\mathcal{E}(\vec{R}, t)=\frac{1}{2 m} \int \frac{\mathrm{d} \vec{p}}{(2 \pi)^{3}}\left(\vec{p}+m \vec{v}_{\mathrm{s}}\right)^{2} G_{\vec{p}}(\vec{R}, t)+\frac{1}{2} \int \mathrm{d} \vec{r}^{\prime} \Phi\left(\left|\vec{R}-\vec{r}^{\prime}\right|\right) \int \frac{\mathrm{d} \vec{p}}{(2 \pi)^{3}} \mathcal{D}_{\vec{p}}^{(1)}\left(\vec{R}, \vec{r}^{\prime} ; t\right)
$$

or

$$
\mathcal{E}=\mathcal{E}_{0}+\vec{j}_{0} \vec{v}_{\mathrm{s}}+\frac{1}{2} \rho v_{\mathrm{s}}^{2}
$$


where

$$
\mathcal{E}_{0}=\frac{1}{2 m} \int \frac{\mathrm{d} \vec{p}}{(2 \pi)^{3}} p^{2} G_{\vec{p}}(\vec{R}, t)+\frac{1}{2} \int \mathrm{d} \vec{r}^{\prime} \Phi\left(\left|\vec{R}-\vec{r}^{\prime}\right|\right) \int \frac{\mathrm{d} \vec{p}}{(2 \pi)^{3}} \mathcal{D}_{\vec{p}}^{(1)}\left(\vec{R}, \vec{r}^{\prime} ; t\right)
$$

By analogy to previous subsections we find

$$
\frac{\partial \mathcal{E}}{\partial t}+\operatorname{div} \vec{Q}=0
$$

The energy flow is given by

$$
\vec{Q}=\left(\mathcal{E}_{0}+\vec{j}_{0} \vec{v}_{\mathrm{s}}+\frac{1}{2} \rho v_{\mathrm{s}}^{2}\right) \vec{v}_{\mathrm{s}}+\frac{1}{2} v_{\mathrm{s}}^{2} \vec{j}_{0}+\vec{\Pi}_{0} \vec{v}_{\mathrm{s}}+\vec{Q}_{0}
$$

where

$$
\begin{aligned}
Q_{0 k} & =\frac{1}{2 m} \int \frac{\mathrm{d} \vec{p}}{(2 \pi)^{3}} p^{2} p_{k} G_{\vec{p}}(\vec{R}, t)+\frac{1}{2 m} \int \mathrm{d} \vec{r}^{\prime} \Phi\left(r^{\prime}\right) \int \frac{\mathrm{d} \vec{p}}{(2 \pi)^{3}} p_{k} \mathcal{D}_{\vec{p}}^{(1)}\left(\vec{R}, \vec{r}^{\prime} ; t\right) \\
& -\frac{1}{2 m} \int \mathrm{d} \vec{r}^{\prime} \frac{\partial \Phi\left(r^{\prime}\right)}{\partial r_{j}^{\prime}} r_{k}^{\prime} \int \frac{\mathrm{d} \vec{p}}{(2 \pi)^{3}} p_{j} \mathcal{D}_{\vec{p}}^{(1)}\left(\vec{R}, \vec{r}^{\prime} ; t\right)
\end{aligned}
$$

The set of equations (15), (16), (18) and (23) form a complete system of two-fluid hydrodynamic equations for superfluid helium-4.

\section{Calculation of the hydrodynamical flows}

In the previous section there was obtained a system of balance equations. These equations are incomplete, because the flows (17), (20) and (25) are unknown. When we have an explicit expression for $G$-function, then finding the hydrodynamical flows is realized by calculating the momentum integrals. In the case of superfluid helium,it is impossible to find the $G$-function. Therefore, we should develop some "indirect" method of finding the flows (17), (20) and (25).

In this article, to find the hydrodynamic flows, we used an explicit expression for the local equilibrium statistical operator. In contrast to paper by Morozov [3] we construct a statistical operator in the system of reference where the condensate is motionless, which provides some simplification.

The local equilibrium statistical operator that describes a superfluid helium in the system of reference where condensate is motionless is as follows

$$
\hat{\varrho}=\exp \left\{\int \mathrm{d} \vec{r} \beta(\vec{r})\left[\Omega(\vec{r})-\hat{H}_{0}(\vec{r})-\vec{u} \hat{\vec{P}}_{0}(\vec{r})-\frac{\mu}{m} \hat{\rho}(\vec{r})\right]\right\} .
$$

In the local frame of reference moving with $\vec{v}_{\mathrm{s}}$ the superfluid component is stopped, then the total current is carried by the normal component.

Therefore,

$$
\vec{j}_{0}=\left\langle\hat{\vec{P}}_{0}\right\rangle=-\frac{\partial \Omega}{\partial \vec{u}} \equiv \rho_{n} \vec{u}
$$

Here, $\rho_{n}$ is the normal fluid density.

Substituting from (27) into (17) we find the momentum density (mass flow)

$$
\vec{j}=\vec{j}_{0}+\rho \vec{v}_{\mathrm{s}}=\rho_{n}\left(\vec{v}_{n}-\vec{v}_{\mathrm{s}}\right)+\rho \vec{v}_{\mathrm{s}}=\rho_{n} \vec{v}_{n}+\left(\rho-\rho_{n}\right) \vec{v}_{\mathrm{s}} \equiv \rho_{n} \vec{v}_{n}+\rho_{\mathrm{s}} \vec{v}_{\mathrm{s}},
$$

where $\rho_{\mathrm{s}}=\rho-\rho_{n}$ is superfluid density.

To find the stress tensor we use a very elegant "scale transformation" method introduced by Bogolyubov [2]. As simple calculation gives

$$
\Pi_{0 i k}=\rho_{n} u_{i} u_{k}+\delta_{i k} P
$$


where $P=\rho \frac{\partial \Omega}{\partial \rho}-$ is pressure.

The final form of a stress tensor is

$$
\Pi_{i k}=\Pi_{0 i k}+v_{s i} j_{0 k}+v_{s k} j_{0 i}+\rho v_{s i} v_{s k}=\rho_{\mathrm{s}} v_{s i} v_{s k}+\rho_{n} v_{n i} v_{n k}+\delta_{i k} P .
$$

To calculate the energy flux we employ an obvious identity [3]:

$$
\langle[A, \hat{S}]\rangle=0,
$$

where $A$ is some dynamic quantities and $\hat{S}$ is an entropy operator, that defined by relationship $\hat{\varrho}=\exp \{-\hat{S}\}$.

Substituting in (31) $A=H$ and using (26), (29) we find

$$
\vec{Q}_{0}=\left(\rho_{n} u^{2}+\frac{\rho \mu}{m}+T S\right) \vec{u}
$$

Finally, expressions for hydrodynamical flows have the form:

$$
\begin{gathered}
\vec{j}=\rho_{\mathrm{s}} \vec{v}_{\mathrm{s}}+\rho_{n} \vec{v}_{n}, \quad \vec{v}_{n} \equiv \vec{u}+\vec{v}_{\mathrm{s}}, \quad \Pi_{i k}=\rho_{n} v_{n i} v_{n k}+\rho_{\mathrm{s}} v_{s i} v_{s k}+\delta_{i k} P, \\
\vec{Q}=\left(\frac{v_{\mathrm{s}}^{2}}{2}+\frac{\mu}{m}\right) \vec{j}+T S \vec{v}_{n}+\rho_{n} \vec{v}_{n}\left(\vec{v}_{n} \cdot\left(\vec{v}_{n}-\vec{v}_{\mathrm{s}}\right)\right) .
\end{gathered}
$$

These hydrodynamical flows coincide with the ones in two-fluid hydrodynamics of Landau [1].

\section{Acknowledgements}

The work was supported by the State Foundation for Fundamental Research of Ukraine (Project No. F25.2/011).

\section{References}

1. Landau L.D. JETF, 1941, 11, 592 (in Russian).

2. Bogolyubov N.N. Preprint UINR, Dubna, 1963 (in Russian).

3. Morozov V.G. Teor. Mat. Fiz., 1976, 28, 267 (In Russian).

4. Bogolyubov N.N., Bogolubov N.N. (jr.) Introduction to the quantum statistical mechanics. Moscow, Nauka, 1984 (in Russian).

5. Svidzynsky A.V. Microscopic theory of superconductivity. Vol. 1. Lutsk, Vezha, 1999 (in Ukrainian).

\section{Мікроскопічна побудова дворідинної моделі для надплинного гелію}

\section{П. Шигорін, А. Свідзинський}

Волинський національний університет ім. Лесі Українки, Луцьк 43025, пр. Волі, 13, Україна

Отримано 24 червня 2009 р., в остаточному вигляді - 30 вересня 2009 р.

Використовуючи систему гайзенбергівських рівнянь руху для нормальної та аномальної кореляційних функцій побудовано дворідинну гідродинаміку для надплинного гелію-4. Виведення базується на розкладі за градієнтами точних рівнянь руху для кореляційних функцій поблизу локальної рівноваги разом з використанням явного вигляду для локально-рівноважного статистичного оператора для надплинного гелію в системі відліку, де конденсат нерухомий.

Ключові слова: дворідинна гідродинаміка, кореляційна функція, надплинний гелій-4, статистичний оператор

PACS: $67.25 . d g$ 
